\title{
Determinación cuantitativa de proteasas de bacterias psicrotróficas aisladas de leche cruda
}

\section{Catherine Márquez G ${ }^{1}$, Karen Piramanrique C $^{1}$, Ana Karina Carrascal C ${ }^{1}$, Bernardo Clavijo D1, Balkis Quevedo1}

\author{
${ }^{1}$ Laboratorio de Microbiología de Alimentos \\ Departamento de Microbiología, Facultad de Ciencias \\ Pontificia Universidad Javeriana \\ Correspondencia: catamarquez@gmail.com \\ Recibido: 06-02-2007 / Aceptado: 08-03-2007
}

\begin{abstract}
Resumen
El uso generalizado de la refrigeración de la leche cruda ha contribuido a mantener la calidad de ésta, pero ha traído como consecuencia la selección de una carga psicotrófica, que durante su desarrollo produce enzimas termoresistentes responsables, en parte, del deterioro de productos de larga vida. Este estudio se diseñó para la cuantificación de la actividad proteolítica de seis microorganismos que corresponden a Pseudomonas fluorescens R12 y R13, Pseudomonas putida R20, Micrococcus luteus R16, Bacillus circulans R5 y Serratia liquefasciens R4, aislados y caracterizados en la leche cruda. Las seis cepas fueron cultivadas en caldo leche al 11\%. Para las curvas de crecimiento, los datos experimentales se ajustaron al modelo de Baranyi. La Pseudomonas putida R20 presentó mejor cinética de crecimiento con $\mu$ máx de $0,1066 \mathrm{~h}^{-1} \mathrm{y}$ un tiempo de duplicación de $6,5023 \mathrm{~h}$. En la determinación de la actividad proteolítica, según Hübner, se estableció que Bacillus circulans $\mathrm{R} 5$ a $5^{\circ} \mathrm{C}$ produjo en la hora 4 de fermentación la mayor cantidad de proteasas $(3,618 \mathrm{UP} / \mathrm{mL})$ en comparación con las demás cepas de estudio. Finalmente se estableció que la temperatura donde existe mayor actividad proteolítica fue $5^{\circ} \mathrm{C}$, que al incrementarse provoca una disminución considerable en la producción de proteasas.
\end{abstract}

Palabras Clave: actividad proteolítica, microorganismos psicotrópicos, modelo de Baranyi, proteasas.

\begin{abstract}
The general use of refrigeration on raw milk has contributed to maintenain its quality, but has induced the selection of psychotropic bacteria which during its growth produces heat-resistant enzymes responsible, in part, for the deterioration of long-life products. This study was designed for the quantification of proteolytic activity in six microorganisms that correspond to Pseudomonas fluorescens R12 y R13, Pseudomonas putida R20, Micrococcus
\end{abstract}


luteus R16, Bacillus circulans R5 y Serratia liquefasciens R4, isolated and characterized of raw milk. The strains were cultured in milk broth $11 \%$ for kinetics profile and the experimental data was adjusted to the Baranyi Model. This Model guaranteed a suitable description of $\mu$ máx, td y Log UFC. Pseudomonas putida R20 showed the best growing profile with $\mu$ máx $=0,1066 \mathrm{~h}^{-1}$ and $\mathrm{td}=6,5023 \mathrm{~h}$. Proteolytic activity was determined according to Hübner and it was established that Bacillus circulans $\mathrm{R} 5$, in a temperature of $5^{\circ} \mathrm{C}$, produced at the $4^{\text {th }}$ fermentation hour the largest amount of proteases $(3,618 \mathrm{UP} / \mathrm{mL})$ with the rest of the used strains. Finally, it was established that the temperature with the largest proteolytic activity was $5^{\circ} \mathrm{C}$ and, as it increases, it causes a considerable decrease in protease production.

Key words: Baranyi model, proteases, proteolytic activity, psychotropic microorganism.

\section{Introducción}

La leche cruda se puede contaminar fácilmente por un amplio grupo de microorganismos ya sea por los diferentes métodos de recolección, almacenamiento y transporte debido a su composición nutricional y a su alto porcentaje de agua dentro de su composición química, razón por la cual el almacenamiento se realiza a bajas temperaturas con el fin de aumentar la vida útil. La leche y los productos derivados de ésta pueden contener microorganismos psicrotróficos, que secretan enzimas como proteasas que se mantienen activas aún después de procesos de pasteurización. Estas enzimas causan degradación en gran parte de las caseínas presentes en la leche, trayendo como consecuencias pérdida de la calidad de quesos, incrementos en la cantidad de nitrógeno y tecnológicamente se produce una reducción de la estabilidad térmica de la leche. Por ende, es indispensable para la industria determinar y cuantificar enzimas producidas por microorganismos que puedan crecer a temperaturas de refrigeración (1).

Considerando lo anterior, este estudio fue diseñado para la cuantificación de extractos enzimáticos de proteasas obtenidos a partir de cinéticas de crecimiento de bacterias psicrotróficas en leche cruda fresca en un sistema automático de fermentación con agitación mínima y bajo condiciones de temperatura controlados $\left(5^{\circ} \mathrm{C}\right)$; a partir de la cual se escogió el microorganismo de mayor actividad proteolítica para ensayos de estabilidad de las proteasas a temperaturas entre 15 y $21^{\circ} \mathrm{C}$ (2).

\section{Materiales y Métodos}

\section{Microorganismos y condiciones de cultivo}

Se seleccionaron 6 cepas de los microorganismos que hacen parte del banco de cepas del Laboratorio de Alimentos de la Pontificia Universidad Javeriana, que corresponden a Pseudomonas fluorescens R12 y R13, Pseudomonas putida R20, Micrococcus luteus R16, Bacillus circulans R5 y Serratia liquefasciens R4, aislados y caracterizados enzimáticamente a partir de 17 cepas con actividad proteolítica medida por halos de hidrólisis a partir de leche cruda (3).

Para la elaboración de las curvas se realizaron preinóculos en un subcultivo con un vial del banco de cepas en $25 \mathrm{~mL}$ de caldo leche (Agua destilada estéril con $11 \%$ de leche incubando en agitación a $21^{\circ} \mathrm{C} \mathrm{du}$ rante 1 hora). Transcurrido ese tiempo se tomaron los $25 \mathrm{~mL}$ y se adicionaron a un elermeyer que contenía $250 \mathrm{~mL}$ de caldo leche para obtener un volumen efectivo de trabajo de $275 \mathrm{~mL}$, permitiendo la aireación a $5^{\circ} \mathrm{C}$ a 1,13g en shaker rotatorio (Infalpar) durante 7 días. Se recolectaron muestras cada 2 horas durante las primeras 12 horas de fermentación y luego cada 12 horas hasta alcanzar la fase estacionaria de crecimiento de las cuales se determinó cada vez el recuento total en placa con agar plate count (Merck) para la determinación de proteínas y la cuantificación de la actividad proteolítica $(3,4)$. 


\section{Recuento de Bacterias Psicrotróficas}

De cada muestra tomada se efectuaron diluciones decimales en agua peptonada al $0.1 \% \mathrm{p} / \mathrm{v}$, para mantener las condiciones isotónicas (2). Para tal efecto se adicionaron $0,5 \mathrm{~mL}$ de la muestra en $4,5 \mathrm{~mL}$ del diluyente para obtener la dilución $10^{-1}$ El procedimiento se realizó por duplicado hasta la dilución $10^{-12}$. A partir de las diluciones se sembraron alícuotas de $0,1 \mathrm{~mL}$ en superficie incubando en aerobiosis a $21^{\circ} \mathrm{C}$ durante 48 horas y se realizó el recuento de microorganismos psicrotróficos $(1,5)$.

\section{Cuantificación de proteínas solubles y actividad proteolítica}

El extracto crudo fue centrifugado a $11319,75 \mathrm{~g}$ (Centrífuga Dynac modelo 420101) durante 10 minutos recuperando el sobrenadante de las muestras para los ensayos de la cuantificación de proteínas solubles y actividad proteolítica, mediciones que se realizaron por triplicado. La cuantificación de proteínas solubles se llevó a cabo mediante la técnica de Bradford (6), realizando lecturas en Multiskan ${ }^{\circledR} \mathrm{MCC} / 340$ a 595nm, utilizando como pro- teína estándar Seroalbúmina Bovina (Sigma) en 0,15M $\mathrm{NaCl}$. Para la cuantificación de la actividad proteolítica se utilizó la técnica del acido tricloro acético, según el método descrito por Hübner, realizando lecturas en Genesys 10 series a 280nm cada una de las muestras por triplicado. Diez (10) Unidades Proteolíticas (UP) equivalen a un aumento de 0.5 en la absorbancia (7).

\section{Efecto de la temperatura en la producción de proteasas}

Se evaluó el efecto de la temperatura, para lo cual se realizaron fermentaciones a $5^{\circ} \mathrm{C}, 15^{\circ} \mathrm{C}$ y $21^{\circ} \mathrm{C}$ con el microorganismo de mayor actividad enzimática y se determinaron proteínas solubles y actividad proteolítica.

\section{Análisis de datos experimentales}

Con los datos obtenidos en las curvas de crecimiento de las cepas de estudio, se realizaron gráficas de log de unidades formadoras de colonias (UFC) contra el tiempo; la interpretación de los parámetros fueron evaluados con el modelo de Baranyi $(3,8)$.

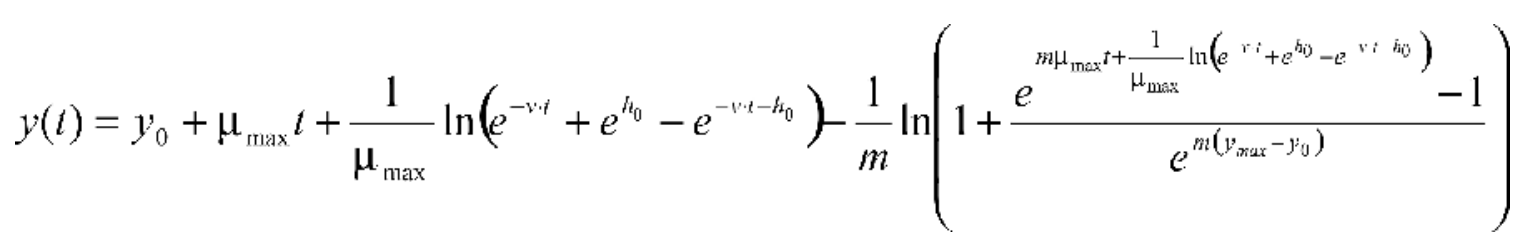

Donde $y(t)$ es el logaritmo de la concentración de células, los parámetros del modelo son $\mathrm{y}_{0}$, ymáx (logaritmo del número inicial y final de células), $\mu$ máx, $\mathrm{m}$ (parámetro de curvatura después de la fase exponencial), $\mathrm{v}$ (parámetro de curvatura antes de la fase exponencial), y h0 (que define el estado fisiológico inicial de las células). La fase log (?) expresada en función h0 es:

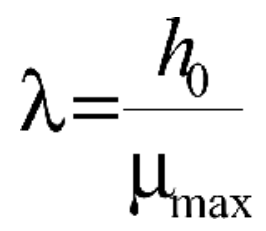

En el modelo según Baranyi, $\mathrm{v}=\mu$ máx y $\mathrm{m}=1$, se reduce el número de parámetros a 4, quedando de la forma:

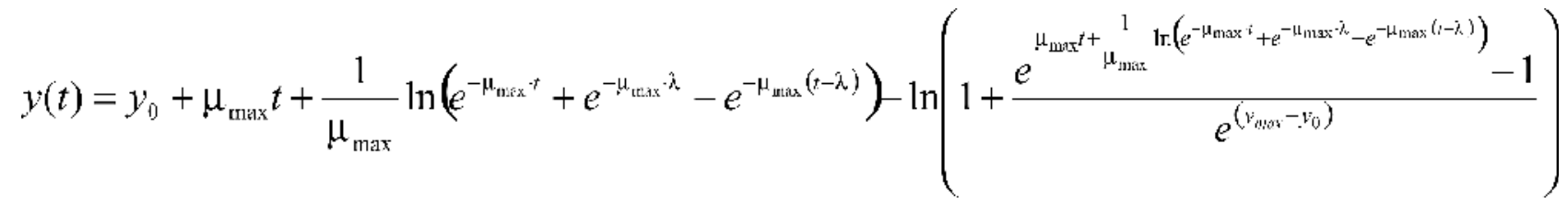




\section{Prueba de Shapiro-Wilk}

Para las determinaciones experimentales de la actividad proteolítica (lecturas o resultados de absorbancias) se analizaron dos grupos de 3 repeticiones, con los cuales se comprobó sí los datos de cada grupo seguían una distribución de frecuencia Gaussiana o normal mediante la prueba de normalidad de Shapiro-Wilk.

\section{Ajuste de los datos experimentales}

\section{Ajuste de las curvas de crecimiento}

Los resultados de los recuentos de cada cepa fueron ajustados para obtener las curvas de crecimiento correspondientes al modelo de Baranyi (GraphPad Prism versión 3.00 para Windows, GraphPad Software Inc, San Diego, USA). Los datos experimentales fueron ajustados al modelo de Baranyi teniendo en cuenta los parámetros cinéticos de $\mu$ máx, td, $\mu, \log \mathrm{UFC} / \mathrm{mL}, \mathrm{R} 2$ y de acuerdo con el error estándar, los límites de confianza y porcentajes asociados en los cálculos de las estimaciones de los datos obtenidos en las curvas.

\section{Análisis estadístico para la actividad proteolítica}

Para este se planteó la determinación de los parámetros cinéticos de la actividad proteolítica, para la cual inicialmente se comprobó la relación matemática entre la actividad proteolítica y el tiempo ajustando la cinética a una reacción de orden cero, lo cual no fue posible ya que los factores de correlación de ajuste a la línea recta se encontraban por debajo de 0,9 . Sin embargo, para Bacillus circulans $\mathrm{R} 5$ a $15^{\circ} \mathrm{C}$ si se cumplió el ajuste a una cinética de orden cero con un R2 de 0,823 (datos no mostrados), teniendo en cuenta que son datos experimentales. No obstante esta cinética no se tuvo en cuenta ya que para el resto de las cepas no se cumplió dicha cinética. $\mathrm{A} 15^{\circ} \mathrm{C}$ se disminuyó considerablemente la actividad proteolítica. Sobre la base de estos resultados se probó una cinética de primer y segundo orden para las demás cepas obteniendo resultados similares a los presentados en la cinética de orden cero. En razón a esto la actividad enzimática se evalúo analizando las unidades proteolíticas en función del tiempo $(8,9)$.

Debido a que se realizaron dos grupos con tres repeticiones para la determinaciones experimentales, para medir la actividad proteolítica, (lectura o resultados de la absorbancia), fue necesario comprobar si existían diferencias estadísticamente significativas entre cada grupo. Para esto se comprobó inicialmente si los datos de cada grupo seguían una distribución de frecuencias Gaussiana o normal, mediante la prueba de normalidad de Shapiro-Wilk (Analytical Software Statistix. 2000).

El análisis de diferencias significativas para los grupos 1 y 2 de cada cepa se realizó mediante la prueba de hipótesis para muestras pareadas (datos con distribución normal) y la prueba no paramétrica de Wilcoxon (datos que no siguen la distribución normal). No se presentan diferencias significativas $(\mathrm{P}>0,05)$ entre los grupos 1 y 2, excepto para aquellos de la cepa Bacillus circulans $\mathrm{R} 5$ a $5^{\circ} \mathrm{C}$ y a $15^{\circ} \mathrm{C}(\mathrm{P}<0,05)$.

\section{Resultados y Discusión}

\section{Curvas de crecimiento}

En la hidrólisis de las proteínas de la leche, por los microorganismos proteolíticos del estudio, fue evidente la clara digestión de la caseína por cambios en el color de la leche inicialmente inoculada, la cual mostró, a lo largo de la fermentación, un proceso de producción de coágulos y reducción del color blanco típico de la leche a un líquido transparente hacia las últimas horas de crecimiento. Este fenómeno es conocido como coagulación dulce de la leche, se precipita la caseína y ocurre sinéresis (2). A pesar de no realizar control del $\mathrm{pH}$ durante las curvas, se realizaron lecturas del mismo (datos no mostrados), que arrojaron valores dentro del rango de $6.6 \pm 2$ en la hora cero hasta un valor mínimo de 5.0 a la hora 120 de fermentación, lo que pudo indicar, de alguna manera, que estos microorganismos producen una proteólisis con escasa acidez. 
La Figura 1 muestra que las cepas Pseudomonas fluorescens R12, Serratia liquefasciens R4 y Micrococcus luteus R16 presentaron un incremento de biomasa de forma rápida, que permitió una adaptación con mayor facilidad al medio, teniendo en cuenta el preinóculo efectuado antes de la fermentación. Mientras que Pseudomonas putida R20, Pseudomonas fluorescens R13 y Bacillus circulans R5 presentaron una pequeña fase de adaptación. Crecimiento que se efectúo de manera constante hasta la hora 24 en las cepas de Micrococcus luteus R16 y Serratia liquefasciens $\mathrm{R} 4$, hasta la hora 60 en la cepa Pseudomonas putida R20, hasta la hora 72 en las cepas Pseudomonas fluorescens R12 y R13, y hasta la hora 96 en la cepa Bacillus circulans R5, momentos en los cuales se delimita la fase exponencial dando inicio a la fase estacionaria.

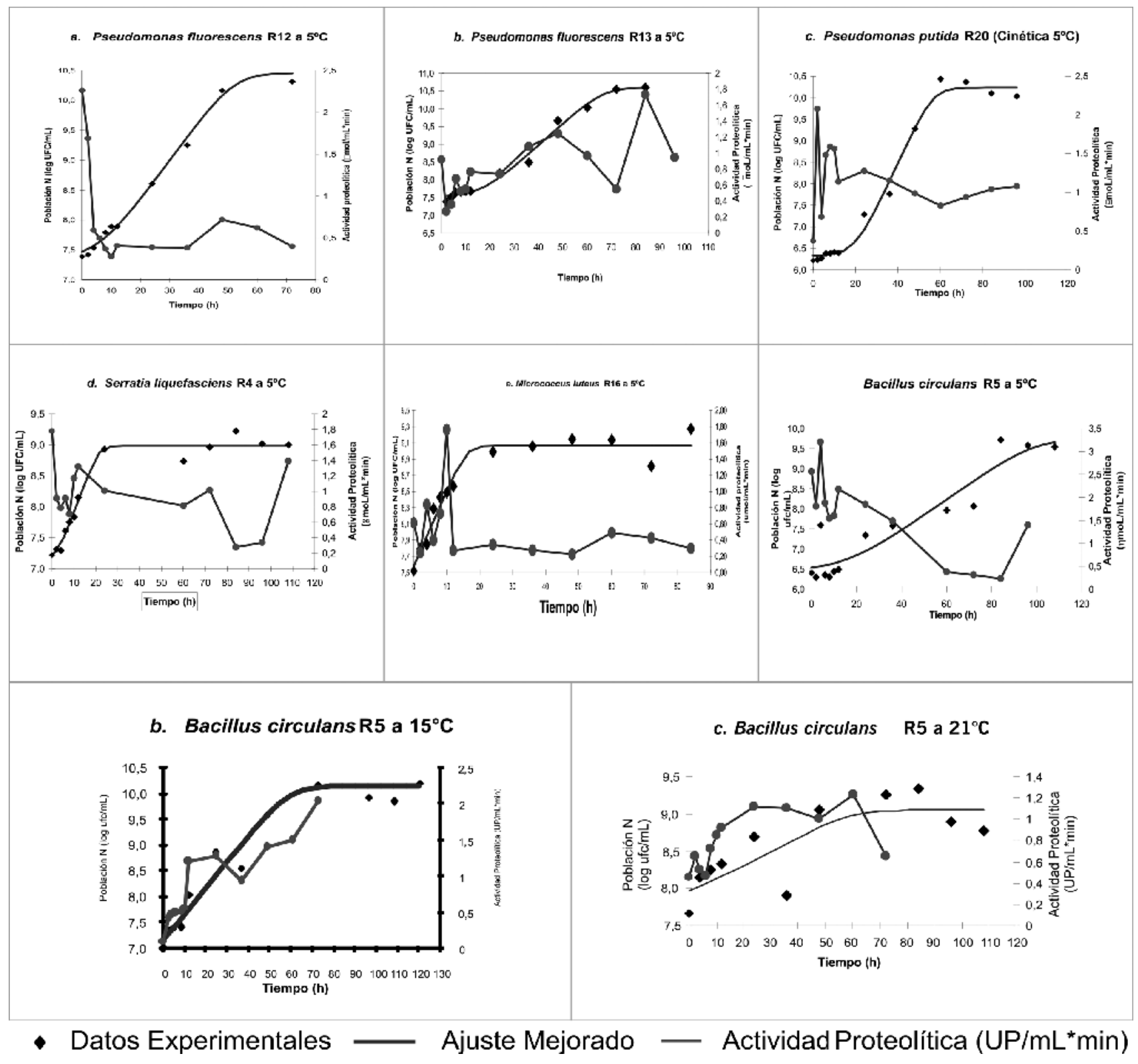

Figura 1. Cinéticas de crecimiento y perfiles enzimáticos de las cepas evaluadas. Fuente: Autores 2004. 
Tabla 1. Tiempos de Duplicación y $\mu$ máx (h-1) de las Cepas. Fuente autores 2004.

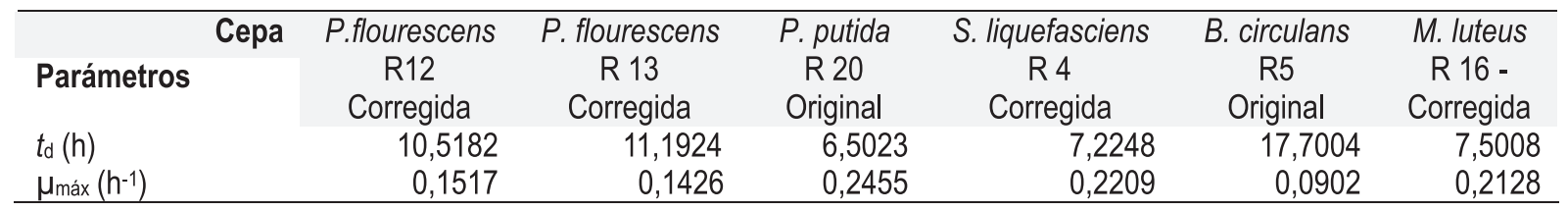

Las cepas Pseudomonas putida R20, Serratia liquefasciens R4 y Micrococcus luteus R16 presentan una muy corta fase exponencial, dada por los tiempos de duplicación de $6,502 \mathrm{~h}, 7,225 \mathrm{~h}$ y $7,501 \mathrm{~h}$ respectivamente y $\mu$ máx de 0,2455h-1, 0,2128h-1 y 0,2209h-1, más altos en comparación con el resto de las cepas evaluadas, Tabla 1. Sin embargo, estos valores de velocidad específica de crecimiento y tiempo de duplicación no representan la posibilidad de una represión catabólica por otros compuestos proteicos de menor complejidad que la caseína, lo que fue demostrado por actividades enzimáticas variables con valores muy altos y muy bajos desde la fase exponencial. De esta forma estos microorganismos incrementaron la actividad enzimática frente a la caseína para continuar con sus funciones degradativas y de crecimiento.

Pseudomonas putida R20 es el microorganismo que presentó mejor cinética de crecimiento, seguido en su orden por Serratia liquefasciens R4 y Micrococcus luteus R16. La selección para la prueba del efecto de la temperatura con base en estos criterios no fue suficiente, es necesario tener en cuenta la actividad proteolítica de las cepas con el fin de elegir no sólo la cepa con mejor cinética de crecimiento sino establecer el mayor productor de proteasas, razón por la cual se escogió Bacillus circulans.

\section{Determinación de proteínas}

\section{Extracción de proteínas celulares solubles}

En la Figura 2 se muestra que la mayor presencia de proteínas durante la curva de crecimiento fue de las cepas Bacillus circulans R5, seguida de Pseudomonas fluorescens R13 y Pseudomonas putida con cantidades de 703,5926, 348,7778 a la hora 8 y $258.407 \mu \mathrm{g} / \mathrm{mL}$ a la hora 24 respectivamente. Estos valores pudieron haberse dado por la presencia de una gran cantidad de proteínas provenientes de la composición del medio leche $11 \% \mathrm{p} /$ $\mathrm{v}$ que son reducidas por la acción enzimática de los microorganismos.

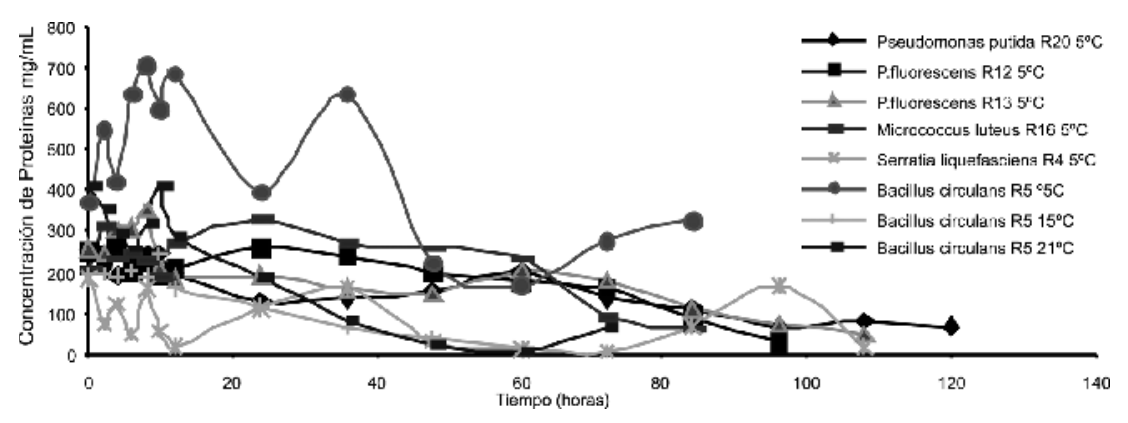

Figura 2. Cuantificación de Proteínas Solubles (Método de Bradford 1976). Fuentes Autores 2004 
Durante las primeras horas de la fermentación la concentración de proteínas de estas 3 cepas es baja debido a que los microorganismos todavía no presentan un aumento significativo de la población y a su vez porque el medio posee sustratos sencillos, fáciles de degradar, que pueden ser utilizados con facilidad por las cepas en su proceso de adaptación al medio. En el caso particular de Bacillus circulans R5, la cepa de mayor actividad enzimática de 3,6177 UP a la hora 4, manifiesta cantidades de proteína de $416,925 \mu \mathrm{g} / \mathrm{mL}$ que corresponde aproximadamente a un poco más de la mitad de la proteína presente en el sustrato

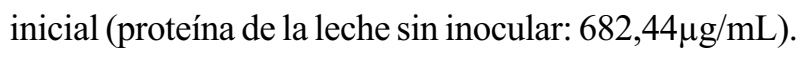

En las cepas Pseudomonas fluorescens R12, Pseudomonas putida R20 y Serratia liquefasciens $\mathrm{R} 4$, presentaron mayor concentración de proteínas entre la hora cero y dos con valores de 255.0741, 215.4444 y $182.8518 \mu \mathrm{g} / \mathrm{mL}$, con lo cual se hizo evidente que la cantidad más alta de proteínas se muestra en las horas iniciales de la cinética, lo que lleva a suponer que la mayor actividad proteolítica se encontrará hacia el final de la fase Log e inicio de la fase estacionaria, teniendo en cuenta que las proteasas en microorganismos psicrotróficos están asociados al crecimiento, Figura 3.

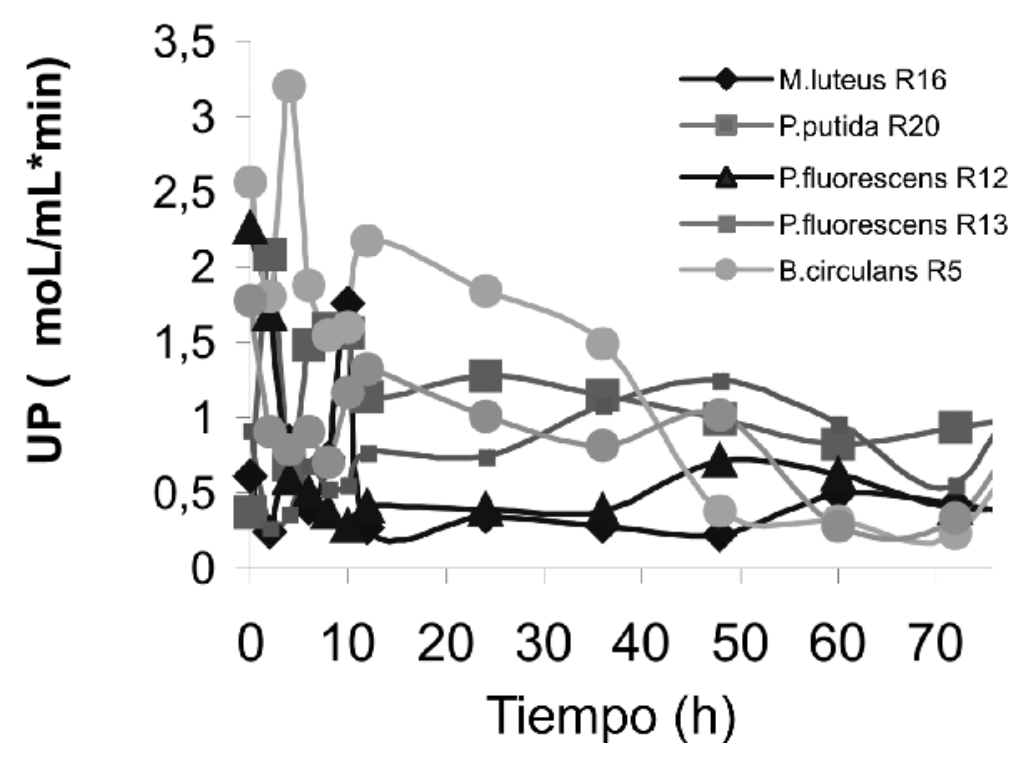

Figura 3. Perfil de Actividad Proteolítica.

Tabla 2. Cepas con Mayor Actividad proteolítica. Fuente: Autores 2004

\begin{tabular}{lcc}
\hline \multicolumn{1}{c}{ CEPA } & UPMáximas $(\triangle \mathrm{ABS})$ & $\begin{array}{c}\text { TIEMPO ÓPTIMO DE } \\
\text { PRODUCCIÓN }\end{array}$ \\
\hline B. circulans R5 & 3,6177 & 4 \\
P. putida R20 & 2.08 & 2 \\
S. liquefasciens R4 & 1.7788 & 0 \\
\hline
\end{tabular}




\section{Actividad proteolítica}

En la Tabla 2 se muestra que Bacillus circulans R5 presentó un valor máximo de actividad en UP de 3.618 a la hora 4, seguida por Pseudomonas putida R20 con 2.08 UP a la hora 2 y en último lugar la cepa de Serratia liquefasciens R4 con una actividad de 1.779 UP en las horas 4,2 y 0 respectivamente.

Al analizar la actividad proteolítica con relación al tiempo de fermentación, es posible observar en las Figuras 1 y 3 , que las cepas presentaron actividad proteolítica desde la hora cero, estos datos son coherentes si se tiene en cuenta que la curva fue iniciada con un preinóculo con una hora de fermentación previa para producir la inducción del sustrato, donde la síntesis de la enzima tuvo lugar en una cantidad significativa, sí se tiene en cuenta que los inóculos utilizados para el estudio presentaban recuentos cercanos a 107UFC/mL. Estos resultados concuerdan con lo reportado por Costa, donde 2 cepas de Pseudomonas $s p$. psicotróficas cultivadas en leche a $7^{\circ} \mathrm{C}$ con concentraciones de crecimiento cercanos a $108 \mathrm{UFC} / \mathrm{mL}$ se produce un incremento notable en la actividad proteolítica desde las primeras horas de fermentación (2).

A pesar de esto se observó en la hora 2 un descenso en la actividad en todas las cepas a excepción Pseudomonas putida R20, datos que indicaron una posible inactivación parcial del complejo enzimático como respuesta fisiológica al inicio del desarrollo en un medio complejo como la leche, lo cual es apoyado con el aumento de la actividad enzimática entre la hora 4 y 6 en las cepas Micrococcus luteus R16, Pseudomonas putida R20 y Bacillus circulans R5, que alcanzan su máximo valor a la hora 10,8 y 4 con $1.7633,1.5933$ y 3.2072 UP respectivamente.

Teniendo en cuenta los resultados de UP cuantificados en las cepas de estudio, la producción enzimática comenzó a incrementarse entre las horas 6 y 8 hasta la hora 24 aproximadamente, por la proteólisis provocada sobre las fracciones proteicas de la leche, las cuales incluyen proteínas del suero aunque en mayor proporción las caseínas, ya que presentan una estructura parcialmente desnaturalizada, por tanto sensibles al ataque enzimático, razón por la cual en la técnica de cuantificación se utilizó como sustrato para la medición de aminoácidos caseína (10).

Según varios autores entre ellos Zaliha y colaboradores (11); probaron la especificidad de diferentes sustratos proteicos naturales y amplia variedad de proteínas modificas y encontraron que las proteasas exhiben su mayor actividad con caseína. De la misma forma Fairbairn y Law, (12); han descrito que las proteasas bacterianas atacan principalmente la k-caseína. Esto produce la desestabilización de las micelas de la caseínas y por lo tanto la coagulación de la leche, gelificación y el desarrollo de sabores amargos. La actividad de las proteasas bacterianas frente a las proteínas del suero es un poco más reducida por ser resistentes a la acción enzimática, atribuible, posiblemente, a la estructura terciara que les proporciona mayor grado de compactación, lo cual las protegería de la proteolísis (2).

La cepa de Pseudomonas fluorescens R13 muestra una actividad enzimática muy baja, que a pesar de incrementarse desde la hora 6, el valor máximo se registra sólo hasta la hora 84, y a lo largo de la fermentación se presentan valores muy bajos en comparación con el resto de los microorganismos analizados. Estos datos concuerdan con lo esperado y con los resultados obtenidos en el estudio de Cujavante (13), donde se observó un halo de proteólisis bastante pequeño $(6 \mathrm{~mm})$, permitiendo deducir que la elección de los microorganismos de acuerdo al perfil mostrado con la técnica de halos fue el adecuado sí se tiene en cuenta igualmente que se buscó cuantificar la actividad para las cepas que presentaran los valores más altos.

En este estudio se corroboró con la cepa de Bacillus circulans $\mathrm{R} 5$ que mostró la mayor actividad y presentó un halo de $30 \mathrm{~mm}$ de hidrólisis, a pesar de observarse que la velocidad de crecimiento (0.0392 h-1) y tiempo de duplicación (17.70horas) son los más bajos en relación con las demás cepas, pero con respecto a la actividad proteolítica, este microorganismo alcanzó los niveles más 
altos de producción y, de alguna manera, constantes a lo largo de la fase exponencial. Razón por la cual, para el análisis de la temperatura, se seleccionó Bacillus circulans R5, a pesar de que según el análisis con los modelos de crecimiento, la selección de la cepa para la cinética a 5,15 y $21^{\circ} \mathrm{C}$ sería Pseudomonas putida R20, por las razones expuestas anteriormente.

Por otra parte, según los resultados de los ensayos realizados utilizando caldo leche al 11\%p/v fue clara la producción de proteasas, pese a esta actividad presentada en todas las cepas, en general no fue alta, lo que indicó que el medio tuvo algún tipo de inhibición para el microorganismo en cuanto a la producción de la enzima. Estudios elaborados por Hübner (10), reportan una actividad de $637 \mathrm{UP} / \mathrm{mL}$ para una cepa del género Bacillus en un tiempo de 12 horas con fermentación continua.

Los resultados para la cepa del mismo género, Bacillus circulans R5 es de 3,6177UP/mL, valor que se encuentra bastante alejado de lo reportado. Estos datos corroboran lo planteado en el estudio realizado por Pereira (9) donde se evaluó el efecto de la caseína en la actividad proteolítica, comparando para tal efecto la utilización de medios nutritivos y un medio con $10 \%$ de leche descremada, pudieron concluir que este último generó un descenso de casi el 88\% de la actividad. Con base en esto es posible deducir que los bajos valores de UP pudieron estar influenciados por este hecho, sin embargo, es relevante tener en cuenta que a nivel de leche el deterioro es notable y bastante significativo.

\section{Efecto de la temperatura en la producción de proteasas}

El efecto de la temperatura sobre la actividad proteolítica fue examinada en el rango de $5^{\circ} \mathrm{C}$ a $21^{\circ} \mathrm{C}$, Figura 4 .

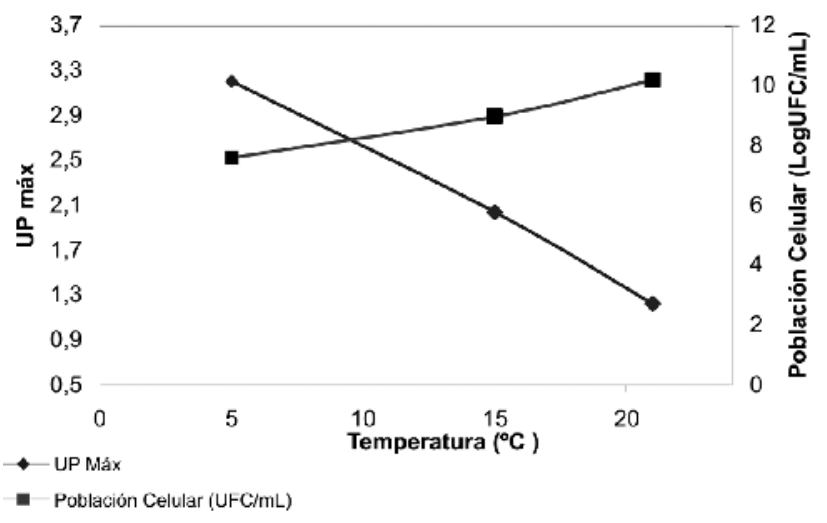

Figura 4. Efecto de la temperatura en la Actividad Proteolítica.

La cepa de mayor actividad proteolítica a $5^{\circ} \mathrm{C}$ fue Bacillus circulans R5. En la Tabla 3 y en la Figura 4 se muestran resultados que apoyan la hipótesis de Costa (2), en la cual las bacterias psicrotróficas sintetizan mayores cantidades de enzima a bajas temperaturas, con el fin de compensar un descenso en la actividad enzimática del metabolismo, el cual le permite el crecimiento y el mantenimiento de las funciones celulares. Tabla 3.

En la Figura 5 se muestran los datos en relación con el crecimiento celular ( $\log$ de $\mathrm{UFC} / \mathrm{mL}$ ) y la producción de proteasas a las diferentes temperaturas probadas. En cuanto al aumento de la población celular, se observó una relación directamente proporcional al aumento de la temperatura de incubación. En el cultivo realizado a $5^{\circ} \mathrm{C}$, se observó que el desarrollo máximo se alcanza a niveles de $5.27 \times 109 \mathrm{UFC} / \mathrm{mL}$ a las 84 horas de cultivo, mientras que en las cinéticas a $15^{\circ} \mathrm{C}$ y $21^{\circ} \mathrm{C}$ el crecimiento celular fue de $3.69 \times 1010 \mathrm{UCF} / \mathrm{mL}$ y $2.14 \times 109 \mathrm{UFC} / \mathrm{mL}$ respectivamente, con lo cual se verificó la capacidad de psicrotolerancia de estos microorganismos (14).

Tabla 3. Actividad Proteolítica a Diferentes Temperaturas de Bacillus circulans R5. Fuente: Autores 2004

\begin{tabular}{cccc}
\hline Temperatura & UP $\left(\boldsymbol{\mu m o l} / \mathrm{mL}^{*} \mathrm{~min}\right)$ & Log UFC/mL & $\begin{array}{c}\text { Tiempo de Producción en } \\
\text { Horas }\end{array}$ \\
$\mathbf{5}^{\circ} \mathrm{C}$ & 3,6177 & 7,5936 & 4 \\
$15^{\circ} \mathrm{C}$ & 2.04 & 8,9778 & 72 \\
$\mathbf{2 1}^{\circ} \mathrm{C}$ & 1,2244 & 10,1891 & 60 \\
\hline
\end{tabular}




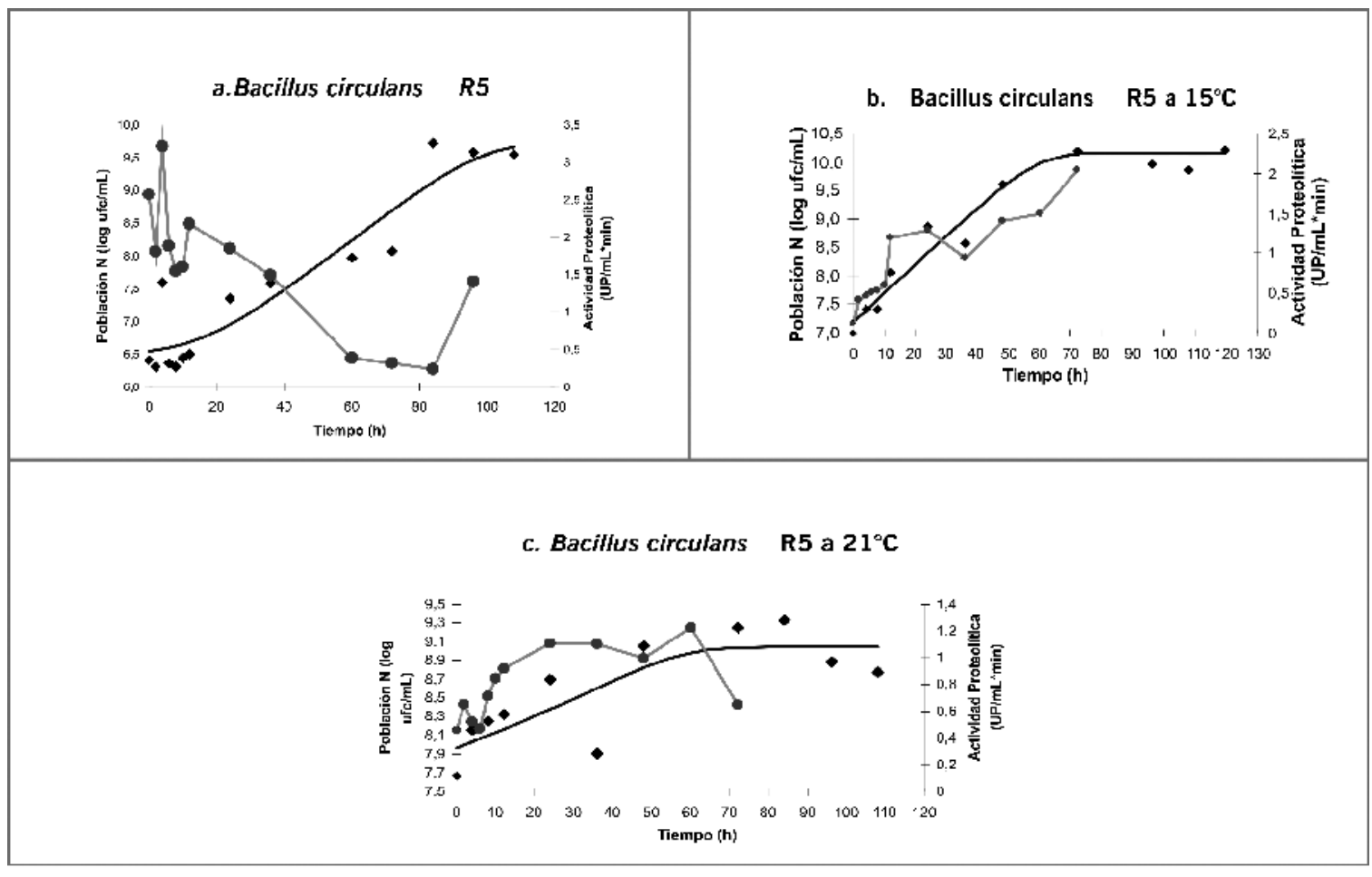

$\checkmark$ Datos Experimentales

Ajuste Mejorado — Actividad Proteolítica (UP/mL*min)

Figura $\mathbf{N}^{\circ}$ 5. Curvas de crecimiento y perfiles enzimáticos de las cepas evaluadas a $5^{\circ} \mathrm{C}, 15$ y $21^{\circ} \mathrm{C}$.

Con referencia a la actividad proteolítica fue posible apreciar una disminución inversamente proporcional al crecimiento microbiano con respecto al incremento de la temperatura, además de presentarse un notorio aumento de la actividad sólo hasta la hora 12 de crecimiento con 1.2033 UP y 0.9222 UP para 15 y $21^{\circ} \mathrm{C}$ respectivamente, mientras que para la cinética de crecimiento de $5^{\circ} \mathrm{C}$ se aprecian valores significativos desde la hora 4, situación que demuestra que la temperatura de incubación influye en la producción de proteasas por unidad de crecimiento en las cepas estudiadas. La temperatura óptima de producción de proteasas por Bacillus circulans R5 es la más baja de las puestas a prueba en el estudio, en contraposición con el efecto de la temperatura que fue de $21^{\circ} \mathrm{C}$.

Los resultados obtenidos en esta investigación permiten anotar, a manera de conclusiones, que los datos de las curvas de crecimiento de las cepas en estudio demostraron que el crecimiento se efectuó de manera constante hasta la hora 24 en las cepas de Micrococcus luteus R16 y Serratia liquefasciens R4, hasta la hora 60 en la cepa Pseudomonas putida R20, hasta la hora 72 en las cepas Pseudomonas fluorescens R12 y R13, y hasta la hora 108 en la cepa Bacillus circulans, momentos en los cuales se delimita la fase exponencial dando inicio a la fase estacionaria con un recuento promedio de $15 \times 10^{9} \mathrm{UFC} / \mathrm{mL}$. Estos datos se ajustaron con el modelo de Baranyi escogido por presentar: mejores valores sensibles, parámetros cinéticos y error estándar que el modelo de Gompertz modificado por Zwietering. Este modelo tiene en cuenta los límites de confianza y porcentajes asociados en los cálculos de las estimaciones de los datos obtenidos experimentalmente en las curvas (15-17).

Con las curvas de crecimiento se logró determinar el tiempo de duplicación (td) de las cepas evaluadas, donde Pseudomonas putida R20, Serratia liquefasciens R4 y Micrococcus luteus R16 tuvieron menores tiempos 
de reproducción de 6,50 horas, 7,50 horas y 10,51 horas respectivamente con respecto a las demás cepas del estudio.

Para la determinación de la actividad proteolítica (UP) se definieron las unidades de actividad enzimática teniendo en cuenta que un incremento de la absorbancia en 0,5 leída a $280 \mathrm{~nm}$ corresponde a $10 \mathrm{UP}$. Al comparar esta definición con los resultados obtenidos de unidades de actividad proteolítica con la curva de calibración de Tirosina, estos valores fueron mayores debido a la adición en el cálculo la tirosina presente en la curva.

El mayor productor de enzimas proteolíticas fue Bacillus circulans R5 con una actividad máxima de $3,618 \mathrm{UP} / \mathrm{mL}$ en la hora 4 . Además, esta cepa sintetiza mayores cantidades de enzima a $5^{\circ} \mathrm{C}$ en comparación con las actividades proteolíticas obtenidas a $15^{\circ}$ y $21^{\circ} \mathrm{C}$. La cuantificación y caracterización de microorganismos psicrótofos contaminantes de leche cruda es un gran avance para determinar la cepa con mayor actividad proteolítica. Para finalizar, una alternativa, para disminuir la actividad proteolítica en la leche, es el tratamiento de ésta con campos eléctricos de alta intensidad que, según datos bibliográficos, han mostrado efectividad en la inactivación de microorganismos y de ciertas enzimas que no es posible inhibir a través de procesos de pasteurización.

\section{Agradecimientos}

Los autores expresan sus agradecimientos a la Universidad Javeriana por la financiación total del proyecto y a todas las personas que hicieron posible la realización de esta investigación. NOV

\section{Referencias}

1. Costa M, Gómez M, Molina 1, Romero A. Growth kinetics and proteases production of pseudomonas fluorescens in raw milk at refrigeration. University Austral of Chile. ALAN. 2001;51:371375.

2. Costa M, Gómez F, Molina H, Simpson R, Romero A. Purificación y caracterización de proteasas de Pseudomonas fluorescens y sus efectos sobre las proteínas de la leche. Instituto de ciencia y tecnología de alimentos, ICYTAL Universidad Austral de Chile. ALAN. 2002;52:160-166.

3. Baranyi J, Mcclure P, Sutherland J, Roberts T. Modeling bacterial growth responses. J Ind Microbiol. 1993;12:190-194.

4. Baranyi J, Roberts T, Mcclure P. A non-autonomous differential equation to model bacterial growth. Food Microbiol. 1993;10:43-59.

5. Robinson R, Phil D. Microbiología lactológica. V1. Ed. Acribia. Zaragoza-España 1987.

6. Bradford M. A rapid and sensitive method for the quantitation of microgram quantities of protein utilizing the principle of protein dye binding. Analytical Biochem. 1976;72:248-254.

7. Hübner U. Entwicklung neuer On-Line- Analysenmethoden zur Steuerung and Regelung von protease fermentationen. Dissertation University of Hanover. 1991.

8. Baranyi J, Robinson T, Mackey B. Predicting growth of Brochotrix thermophacta at changing temperature. Int J Food Microbiol. 1995;27:61-75.

9. Pereira C, Barreto M, San Romão M. Evidence for proteolytic activity and biogenic amines production in Lactobacillus curvatus and L.homohiochii. Inter J Food Microbiol. 2001;68:211-216.

10. Hübner U, Bock U, Schügerl K. Production of alkaline serine protease subtilisin Carlsberg by Bacillus licheniformis on complex medium in a stirred tank reactor. App Microbiol Biotechnol. 1993;40:182-188.

11. Zaliha R, Razak N, Ampon K, Basri M, Wan M, Yunus W, Salleh A. Purification and characterization of a heat-stable alkaline protease form Bacillus stearothermophilus F1. Appl Microbiol Biotechnol. 1994;40:822-827.

12. Fairbairn D, Law B. Proteinases of psychotropic bacteria: their production, properties, effects and control. J Dairy Res. 1986;53:457-466.

13. Cujavante A, Rico M, Clavijo B. Aislamiento y caracterización de microorganismo psicotrofos con actividad proteolítica en la leche cruda. Tesis de pregrado. Pontificia Universidad Javeriana. Facultad de Ciencias, Bogotá. 2002.

14. Hoshino $\mathrm{T}$, Ishizaki $\mathrm{K}$, SakamotoT, Kumeta H, Yumoto J, Matsuyama H, Ohgiya S. Isolation of a Pseudomonas species from fish intestine that produces a a protease active al low temperature. Lett Appl Microbiol. 1997;25:70-72.

15. Baranyi J, Roberts T. A dynamic approach to predicting bacterial growth in food. Int J. Food Microbiol. 1994;23:277-294.

16. Baranyi J, Roberts T. Mathematics of predictive food microbiology. Int J Food Microbiol. 1995;26:199-218.

17. Zwietering M, Jonegenburger I, Rombouts F, Van't Riet K. Modelling of the bacterial growth curve. Appl. Env. Microbiol. 1990;56:1875-1881.
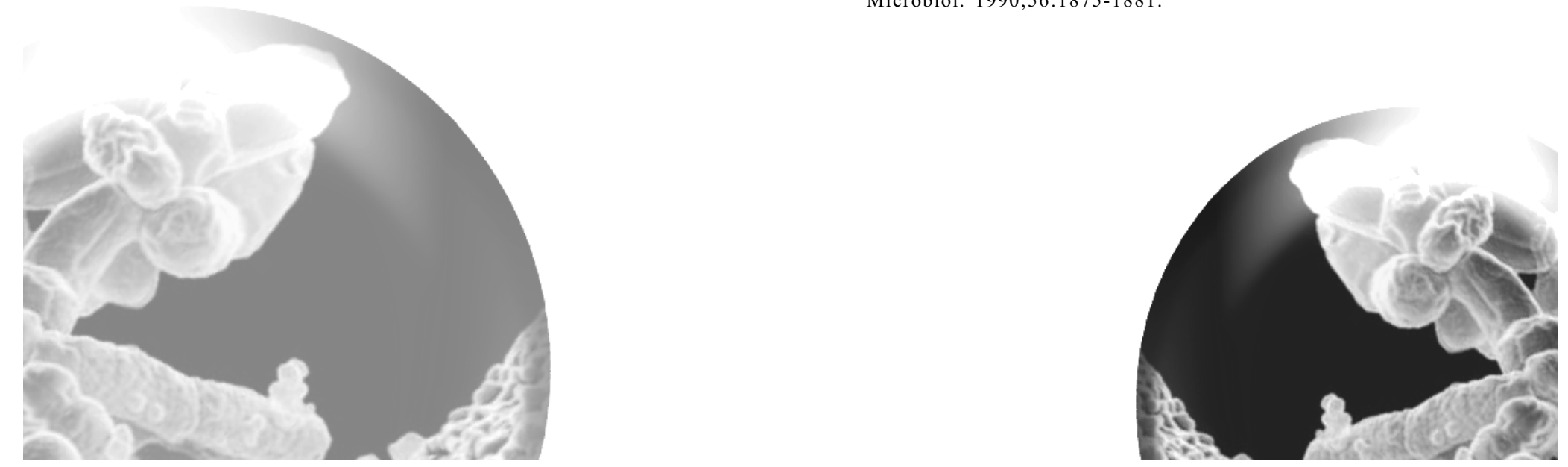